\title{
Update on acute promyelocytic leukemia in the pediatric population
}

\begin{abstract}
Acute promyelocytic leukemia (APL) has shown differences in incidence around the world, it has a characteristic molecular, pathogenic and clinical development mechanism. Despite being a low incidence acute myeloid leukemia (AML) compared to other AML and having a good response to therapy, it continues to be of interest due to the toxicities of its current treatment, to mutations that have shown resistance to those drugs, and to the few possibilities of alternative treatment with all-trans retinoic acid (ATRA) and anthracyclines in the pediatric population. Furthermore, the description of new mutations that originate APL produced by LPA generates new interest in studies that may shed light on their effects on the disease's incidence, prognoses, and response to treatment. This article aims to review different studies that describe findings of APL in terms of incidences, treatments, side effects, survival, especially in the pediatric population. Different studies propose the use of ATRA - arsenic trioxide (ATO) as the first line of treatment, however, few have been carried out in the pediatric population and the use of ATO is not recommended in children under 5 years of age. On the other hand, the differentiation syndrome (DS) has been described, which occurs in patients treated with ATRA, and requires careful monitoring and control.
\end{abstract}

Volume 9 Issue 5 - 202|

\author{
Adriana Ruiz-Rodriguez,' Lucia Obando- \\ Madrigal, ${ }^{2}$ Ana Lucia Mateus-Vargas ${ }^{3}$ \\ 'Hospital Dr. Carlos Luis Valverde Vega, CCSS, Costa Rica \\ ${ }^{2}$ Laboratorio de Pruebas de Paternidad, CCSS, Costa Rica \\ ${ }^{3}$ Área de Salud Alajuela Sur, CCSS, Costa Rica
}

Correspondence: Ruiz-Rodríguez Adriana, Hospital Dr. Carlos Luis Valverde Vega, CCSS, Costa Rica, Email adriruro@gmail.com

Received: September 17, 2021 | Published: October 01, 2021

Keywords: pediatric leukemias, acute promyelocytic leukemia, differentiation syndrome, arsenic trioxide, anthracyclines, all-trans retinoic acid

\section{Introduction}

Acute promyelocytic leukemia (APL) causes between 5\% and up to $15 \%$ of all myelocytic leukemias in pediatric age, although the highest incidence is observed in Latin American countries, such as Brazil, Mexico, Peru, and Venezuela, where it is reported higher than $20 \%$; but in general terms, it is considered rare in children. ${ }^{1-3}$

Specifically, the morphology corresponds to the M3 or M3v (hypogranular variant) subtype of the Franco-American-British (FAB) classification. $95 \%$ of APL cases are characterized by a translocation between chromosome 15 and $17 \mathrm{t}(15 ; 17)(\mathrm{q} 22 ; \mathrm{q} 21)$ and the fusion between the PML gene and the retinoic acid receptor alpha gene in chromosome 1, giving rise to the formation of the PMLRARa protein. This protein causes the arrest of differentiation in the promyelocytic stage, which leads to an accumulation of leukemic cells similar to promyelocytes in the bone marrow. In $5 \%$ of APL, other translocations such as $\mathrm{t}(5 ; 17)$ or $\mathrm{t}(11 ; 17)$ are found, but they generate a similar pathophysiological effect. The resulting PMLRARa hybrids retain most of the functional domains of the parental proteins and act as dominant-negative oncogenic products, interfering with the functions of the retinoid pathway and PML. ${ }^{2,4,5}$

The diagnosis of the disease can be carried out with the confirmation of the $t(15 ; 17)$ translocation and the PML-RARa protein, although there are other possible translocations. Auer bodies, linear azurophilic granules within the cytoplasm of blasts composed of myeloperoxidase may also be present. The immunophenotype shows CD13, CD33, CD17 and, myeloperoxidase positivity conceals with a high sidescatter. While there is an absence or decreased expression of CD34, HLA-DR, CD10, CD11a, CD11b, CD17, and CD18. The CD56 marker is associated with a poor prognosis in $10 \%$ of patients. ${ }^{6}$

Recent studies suggest that obesity increases the risk of APL, but to date, there are no specific risk factors that have been associated with children. ${ }^{6}$ This review aims to discuss mutations, current treatment and, clinical trials, the effect on coagulation, and some clinical cases of APL in the pediatric population.

\section{T $(I 5 ; \mid 7)$ translocation and PML RARa protein}

The RARp gene is a ligand-dependent transcription factor that binds to retinoid X receptors (RXR) to form transcriptionally active heterodimers. The response elements to retinoic acid (RARE) are found in genes whose function is essential in processes of growth, differentiation, survival, and cell death. ${ }^{7}$

The PML protein plays an important role in the processes of cellular homeostasis and tumor suppression mechanisms, response to viral infections, stress, angiogenesis, differentiation, and maintenance of genome stability.

PML-RAR is said to retain the ability of RAR to bind to elements that respond to retinoic acid and dimerize with the retinoid receptor $\mathrm{X}$ protein, but it inhibits the normal values of gene transcription regulated by these elements, leading to repression of RAR target genes and blocks differentiation at the promyelocyte stage. Another described mechanism indicates that PML-RAR prevents PML nuclear bodies' formation, which usually leads to the activation of p53 tumor suppressor pathways and induces cellular senescence in stress situations. Therefore, PML-RAR causes failure of p53 activation with decreased cell death and increased self-renewal. Therefore, both mechanisms (failure of myeloid differentiation and uncontrolled proliferation) lead to the development of leukemia. ${ }^{7}$ Even in the absence of $\mathrm{t}(15 ; 17)$, rearrangements can occur leading to the production of PML-RARa transcripts. Hence, PLM-RARa transcripts are considered a more specific diagnosis than just translocation.?

The RARp breakpoint is almost always in intron 2 of the RARp gene, however, the PML breakpoint can occur in 3 different groups, called bcr, which can generate isoforms of the PML / RARA gene. 
The most common breakpoint in APL occurs in intron 6 that generates the bcr1 isoform. The second most common is the one that occurs in intron 3 (bcr3), and exon 6 (bcr2). The prevalence of each bcr varies by ethnicity. It has been seen that bcr3 is more common in children than in adults. In adults, bcr 2 is associated with higher rates of relapse, while ber3 with higher leukocytosis and worse prognosis, but still lacking studies to be considered in risk stratification. ${ }^{7}$

\section{Other mutations}

In addition to the PML gene, in $1 \%-2 \%$ of cases, APL is due to rare variants where the RARA gene binds to others, such as NPM1, NUMA1, PLZF, STAT, and STAT5b, ZBTB16, PRKAR1, FNDC3B, PRAR1A, NABP1, GTF2I, IRF2BP2, TBL1XR1, BCOR, FIP1L1. ${ }^{8-10}$

However, there are also studies where it is shown that certain APL patients lack the mutation that involves the RARA gene so that a retinoic acid receptor (RARB) gene binds to other genes, the most common being TBL1XR1-RARB. The effect of this mutation is the same, blocking cell differentiation and inducing proliferation. It has been seen that ATRA and ATO treatments have a partial effect on this mutation. ${ }^{10}$ The mutation in the FMS-like tyrosine kinase gene (FLT3) has been observed in up to $40 \%$ of pediatric cases. This mutation has also been described in other myeloid leukemias and gives them a poor prognosis, but its use in APL as a prognostic indicator is still controversial.

\section{Thrombosis and bleeding in APL}

APL is characterized by complications associated with coagulopathy, where both bleeding and thrombotic events can occur There are 2 mechanisms associated with this, on one hand, there is profound coagulopathy compared to disseminated intravascular coagulation (DIC), induced by overexpression of tissue factor, and hyperfibrinolysis, induced by the expression of various coagulation factors in LPA's blasts, such as cysteine protease, tissue plasminogen activator, urokinase-like plasminogen receptor activator, and annexin II; which causes a state of hypercoagulability and an increase in fibrinolysis. In addition, there is an increase in elastase and depletion of $\alpha 2$-antiplasmin and a decrease in the inhibitor of thrombin-activated fibrinolysis. In DIC caused by LM3 the levels of protein C, protein S and, antithrombin III are normal. ${ }^{1,6}$

In the initial phase of APL, most patients present with bleeding in the form of bruises, petechiae, epistaxis, and menorrhagia. Hence, the main complication and cause of premature death in clinical trials are hemorrhagic complications in the central nervous system and lungs, mainly during the first 15 days after diagnosis. To avoid this, it is recommended to provide timely transfusion support, where platelets, fibrinogen and, other coagulation factors are administered prophylactically. ${ }^{1,2}$ And as in adults, a high leukocyte count (> $10.0 \times 10 / \mathrm{L}$ ) is considered a risk factor for premature death secondary to hemorrhage in pediatric patients. ${ }^{1}$ Despite prophylaxis, the rates of premature death from bleeding events remain the same. ${ }^{6}$

In the study carried out by de Albuquerque-Antunes et al., ${ }^{1}$ it was determined that $89 \%$ of the patients had hemorrhagic manifestations, mainly mucocutaneous bleeding. Concerning laboratory tests, it is common to find prolonged prothrombin time (PT) and activated partial thromboplastin time (PTT), low fibrinogen, high D-dimer and, thrombocytopenia. ${ }^{6}$

For its part, thrombosis is not as well recognized, and at the same time, it is underestimated, possibly concealed by the other more common bleeding complications. Risk factors for APL thrombosis include leukocytosis $\left(\gg>30 \times 10^{9} / \mathrm{L}\right)$, signs of DIC, presence of the expression of FLT3-ITD, and the bcr3 PML-RARA isoform, as well as the expression of CD2 and CD15, microgranular subtype, and low fibrinogen levels. Thrombosis can occur at any time during treatment, either before, during, or after induction. ${ }^{1}$ Furthermore, ATRA can induce a hypercoagulable state after correction of the fibrinolysis syndrome and this induces the expression of adhesion molecules. ${ }^{1}$

Regarding early death, it has been shown that high white blood cell counts, high blast counts in peripheral blood, presence of M3v, and belonging to black ethnicity, do not correlate with the risk of early bleeding or death. ${ }^{8}$ However, in the study carried out by De Azevedo et al., ${ }^{11}$ it was found that patients with early death have a higher white blood cell count, a higher promyelocyte count in peripheral blood, and a higher platelet count than in those patients who have not had an early death. Early death is defined as those deaths that occur within the first 30 days after diagnosis. ${ }^{11}$

Furthermore, it was found that a white blood cell count greater than $10 \times 10^{9}$ cells $/ \mathrm{mL}$, a promyelocyte count at the beginning of the disease above $20 \times 10^{9}$ cells / $\mathrm{mL}$, the M3v subtype (hypogranular variable), and serum creatinine above $0.7 \mathrm{mg} / \mathrm{dL}$, predisposes patients to early death, as well as the presence of coagulopathies. The FLT3 mutation has also been found in more than $50 \%$ of patients with early death. ${ }^{9,11}$ One of the risks involved in presenting $\mathrm{M} 3 \mathrm{v}$ is that its diagnosis can initially be mistaken for Acute Monocytic Myeloid Leukemia, which makes the diagnosis and treatment start later., ${ }^{9,11}$

Among the main causes of death in children, especially in children with obesity, including early death, which is the main one, are hemorrhages in the central nervous system, intracranial hemorrhages, and secondary infections. In different studies, it has been found that early death occurs in $9.7 \%-20 \%$ of patients. However, it is presumed that the early initiation of ATRA / ATO treatment reduces the risk of early death, except for patients with APL $t(11 ; 17)$ (q23q21) that is related to resistance to those agents. ${ }^{1,9,12}$

\section{Treatment and prognosis}

The PML / RARA fusion causes the cell maturation of myeloid progenitors to stop in the promyelocyte state. An early diagnosis and initiation of treatment prevent bleeding complications and even death. ${ }^{8}$ The PML / RARA protein plays a very important role in the pathogenesis of the disease, but it is also an important molecular marker for the monitoring of minimal residual disease (MRD) in the prognosis of this disease. ${ }^{12}$

\section{All-trans retinoic acid (ATRA)}

ATRA is a vitamin A analog, which combined with anthracyclinebased chemotherapy is the standard treatment. Its effectiveness is greater than $80 \%$, although there have been reports of effectiveness of up to $94 \%$ of patients reaching complete remission, and 5-year disease-free survival (DFS) of $80 \%$. It is estimated that $10 \%-20 \%$ of children with acute promyelocytic leukemia will relapse. ${ }^{11,12}$

ATRA binds to the PML / RARA fusion, promotes the degradation of this oncoprotein, and activates the transcription of genes responsible for cell differentiation, thereby giving continuity to granulocyte maturation, and finally to apoptosis. ${ }^{7,8,13}$

Chemotherapy based on the combination of ATRA with anthracycline in induction therapy in a newly diagnosed pediatric population with APL has been widely shown to be effective in achieving complete remission in more than $90 \%$ of cases, and it is considered difficult for another type of treatment to reach these levels 
of effectiveness. ${ }^{12}$ However, due to the high cumulative anthracyclines dose that is administered to keep the risk low, other drugs such as ATO have been studied, which in combination with ATRA, have shown high effectiveness in adults. ${ }^{12}$

\section{Arsenic trioxide (ATO)}

Treatment with ATO began when the patient had a relapse, and its effectiveness in achieving a second remission reached $85 \%$. However, this drug has represented a new era in APL, which is why it is also being used as the first-line treatment, reaching $86 \%$ effectiveness. ${ }^{8,13}$

ATO causes degradation of all molecular species that contain LPA, promotes apoptosis of LPA cells, has pro-oxidative effects and, damages structural proteins. ${ }^{7}$ ATRA and ATO combination allows the proteasomal degradation of the PML-RARA oncoprotein via ubiquitination and finally reactivates the transcription of RARA target genes. Other mechanisms of action include the recruitment of caspases, the production of reactive oxygen species, and the formation of PML-nuclear bodies, the architecture of which is broken by the PML-RARA fusion protein, with critical consequences for normal cellular functions, such as self-renewal. ${ }^{14}$

Despite the good remission results obtained with ATRA treatment, severe side effects have been seen both in the short term (thrombocytopenic purpura, pancarditis and, pulmonary infiltrations) and in the long term (heart disease, secondary neoplasms), which hinders a favorable long-term progression, especially in children and reduce their life expectancy. ${ }^{7,8}$ On the other hand, ATO has been associated with risk for the development of severe infections and secondary leukemias. ${ }^{7}$ Today, a chemo-free treatment, with an ATRA- ATO approach, is the first-line treatment in low-risk APL patients. With proper treatment, most patients are cured, however, approximately $5 \%$ of cases are resistant to standard therapy, and 5\%$10 \%$ of cases relapse and can generate resistance. ${ }^{7}$

Studies conducted in patients treated with ATRA and ATO haven't shown many side effects. However, the ATRA syndrome has been described which presents fever, dyspnea, pleural and/or pericardial effusion, pulmonary infiltrates on X-ray films, and weight gain. The differentiated syndrome is also described when the patient presents fever, cough, dyspnea, peripheral edema, weight gain, retention of pleural fluids, and pulmonary infiltrates. In the case of long-term adverse effects, aseptic bone necrosis and cerebrovascular accidents have been found in less than $10 \%$ of cases. ${ }^{9,12}$ Another complication has also been described in children and adolescents treated with ATRA, known as Pseudotumor cerebri, due to an increase in the excretion of choroidal secretion in the cerebrospinal space. ${ }^{12}$

To avoid the possible adverse effects of ATRA in the pediatric population, studies have been carried out in which treatment protocols are applied that include doses of ATRA lower than the conventional dose. Park et al. ${ }^{15}$ conducted a study with 76 patients under 18 years of age, $67.1 \%$ of them were administered the conventional dose of 45 $\mathrm{mg} / \mathrm{m}^{2} / \mathrm{d}$ and the remaining $32.9 \%$ received a low dose of $25 \mathrm{mg} /$ $\mathrm{m}^{2} /$ day. They observed that the incidence rate of RA Syndrome was higher in patients who received the conventional dose $(64.7 \%)$ and for patients who received a low dose the incidence of the syndrome decreased to $36 \%$. Likewise, a higher incidence of headaches was recorded in patients who received the conventional dose, but there was no statistically significant difference between the two groups. They found no statistically significant differences between the rates of complete remission, the relapse rates, and the survival rates of both groups. ${ }^{15}$

\section{ATO in the pediatric population}

In pediatric patients, the use of ATO decreases exposure to a high cumulative dose of anthracycline, and therefore reduces some longterm adverse effects. Furthermore, it also has the potential to increase efficacy in patient populations with a higher prevalence of high-risk disease, ${ }^{16}$ however, ATO is not recommended in patients under 5 years of age. ${ }^{12}$

Kutny et al. ${ }^{17}$ published the results of the non-randomized phase III study AAML0631, in which 101 patients from 2 years to 21 years were treated with ATO during the consolidation phase, to verify its safety and tolerability. The use of ATO allowed a decrease of approximately $40 \%$ in the cumulative dose of anthracycline without compromising the survival of standard-risk APL patients. The liver toxicity produced by the ATO was minimal and there were no serious cardiac events due to its administration.

For patients who survived to receive ATO in consolidation phase 1 , the three-year disease free survival was $93 \%+/-6 \%$ in standardrisk patients and $89 \%+/-12 \%$ in high-risk patients. For all patients, the three-year overall survival was $94 \%+/-5 \%$ and the three-year event-free survival was $91 \%+/-6 \%$. The relapse rate at three years was $4 \%+/-4 \% .^{17}$

Zhang et al. ${ }^{18}$ carried out a randomized prospective study with 66 children aged 14 years or younger who were diagnosed with APL, with which they set out to determine whether the combination of ATRAATO is safe and effective in pediatric APL and whether cytarabine chemotherapy can be skipped when two courses with ATO are added.

In this study, 65 patients entered complete hematological remission, only one of the patients who did not receive cytarabine relapsed. After a median of 35 months of follow-up, the event-free survival was 97.3 $+/-2.7 \%$ and the total survival was $100 \%$. Arsenic levels measured in patients' plasma, urine, nails, and hair indicated that there was no significant accumulation of arsenic after ATO administration had been discontinued for 12 months. ${ }^{18}$

Kutny et al. ${ }^{19}$ conducted a cohort study in which they included 83 patients under 18 years of age with a diagnosis of APL. In the case of the consolidation phase, patients aged 15 years and older were randomized to receive 2 cycles of ATO or standard treatment. In total, 12 patients were randomized with ATO and 9 patients were given standard consolidation therapy. The risk of relapse at 5 years was significantly lower in patients who received ATO than in those who did not ( $0 \%$ vs. $44 \%)$. However, the authors acknowledge the inherent limitations of these subgroups being small.

\section{Resistance to ATRA and ATO treatment in APL}

Resistance to treatment with ATRA-ATO is of concern and prompts new research for novel therapies for APL. Resistance to ATRA-ATO may be due to genetic mutations that cause an amino acid substitution in the ligand-binding domain of RARp (LBD) and the PML-B2 domain of PML-RARA. Several mutations have been described in these domains, however, they cannot explain the majority of clinically found resistance to ATO. ${ }^{7}$

When a patient relapses or becomes resistant to treatment, hematopoietic stem cell transplantation appears to be the only option to achieve a cure. However, many studies have been dedicated to the search for other molecules within retinoids, such as the currently studied tamibarotene. In addition, they have focused on identifying 
other mechanisms of action and new compounds that act in synergy, to prevent resistance to retinoids and ATO.

Inhibitors of HDACs, such as sodium butyrate, valproic acid, and trichostatin A, have been used in combination with ATRA. Antibodies anti-CD33-conjugated with calicheamicin, gentuzumab ozogamicin (GO)have also been used because they are considered effective against LPA cells with high expression of CD33., ${ }^{72}$ Analysis of the response to treatment in aberrant mutations has been carried out, however, no significant differences have been found in the evolution concerning patients with $\mathrm{t}(15 ; 17){ }^{9}$

In the retrospective study carried out by Czogala et al. ${ }^{9}$ in 41 patients, one patient $(2.4 \%)$ had a relapse after having been in remission for 17 months. This patient was treated with ATO, and due to the non-response to this treatment, he had to be transplanted with hematopoietic stem cells and continued with ATO. Finally, and due to the progression of the disease, the patient died.

On the other hand, studies have also been carried out in search of alternatives to the treatment that can increase the effect of ATRA. Hassibe et al. ${ }^{20}$ studied the non-T cell activation linker (NTAL) gene. NTAL is a membrane protein lipid expressed in normal B cells, NK cells, mast cells, and monocytes. Furthermore, it has been found in the cells of some AML, such as APL. In this study, it was found that NTAL is important in cell survival, and is associated with high white blood cell counts. The depletion of this lipid in cells decreases cell viability, affects cell proliferation, and increases sensitivity to drugs such as ATRA. ${ }^{20}$

\section{Hematopoietic stem cell transplantation}

In hematopoietic stem cell transplantation, good prognoses have been reported in the second remission in adult patients. Regarding pediatric patients, Yamamoto et al. ${ }^{21}$ conducted a retrospective study analyzing data from pediatric transplanted patients in Japan. They found that transplant patients without having presented a remission had the worst survival rates (5yOS) at 5 years. However, they did not find significant differences in this same index, and the disease-free 5 -year index (5yDFS), when the transplant occurred after the first, second, or third remission.

On the other hand, it was found that, although an autologous transplant is recommended in second remission, there were no significant differences in the mortality rates related to transplantation and the incidence of relapse, between autologous and allogeneic transplants. ${ }^{21}$

\section{Oral arsenic therapy}

The Realgar-Indigo Naturalis (RIF) formula is an arsenic compound that comes from Chinese medicine, it was approved by the Food and Drug Administration of that country for the treatment of APL in adults in 2009. The pharmacokinetic profile plus the favorable outcome of this oral formulation has led to lower liver and cardiac toxicities and protocols for the post-induction phase that do not require hospital admission, saving costs for families and health systems, as well as favoring the psychological condition of patients. ${ }^{14}$

Yang et al. ${ }^{22}$ conducted a prospective and randomized study with 82 patients aged 16 years or younger, to determine whether ATO applied intravenously together with ATRA and anthracycline-based chemotherapy can be substituted by oral RIF in the treatment of Pediatric APL.

For both groups of patients, overall survival at 5 years and event-free survival at 5 years of $100 \%$ was recorded. The rate of treatment-related adverse events was similar in both groups, except for the incidence of infection and fever of unknown origin, which were significantly lower in the group that received RIF. The mean accumulated days of hospitalization during induction and consolidation therapies for standard-risk patients was $67.8+/-22.4$ in the group that received ATO and $43.9+/-19.3$ in the group that received RIF; in high-risk patients, $68.1+/-19.6$ days accumulated for the group that had ATO as part of their therapy and $48.1+/-18.6$ for the group that received RIF during their therapy. ${ }^{22}$

Zheng et al. ${ }^{23}$ published an investigation carried out with 186 patients with APL under 18 years of age, who were stratified into two groups: standard risk and high risk. The standard-risk group received RIF or ATO with ATRA during the induction and consolidation phases, and the high-risk group received anthracyclines, in addition to the drugs already mentioned. Patients received ATO or RIF depending on the availability of these agents in the hospital where they were being treated.

The 2-year overall survival rate was $99 \%$ for the standard-risk group and $95 \%$ for the high-risk group. The event-free survival rate at 2 years was $97 \%$ for the standard-risk group and $90 \%$ for the highrisk group. There was no significant difference between both groups for these indicators. Regarding the cumulative incidence of relapse at 2 years, this was $2 \%$ for the standard-risk group and $6 \%$ for the highrisk group..$^{23}$

Arsenic concentrations in urine, plasma, hair, and nails of 34 of the patients who participated in the study were quantified. The results obtained showed that the retention of arsenic did not result in a significant accumulation of it at 6 months after cessation of treatment. Common signs of chronic arseniasis were not observed, such as cardiovascular events, chronic kidney failure, diabetes, or neurological dysfunction. ${ }^{23}$

\section{Incidence worldwide}

Jastaniah et al. ${ }^{24}$ carried out a retrospective study in which they recruited patients 14 years of age or younger from nine health centers in Saudi Arabia. Of the 207 patients who were diagnosed de novo with AML between January 2005 and December 2012, 32 of them were diagnosed with APL, which is equivalent to $15 \%$, with a median age at diagnosis of 10.4 years.

Park et al. ${ }^{15}$ conducted a retrospective investigation in which they found a $9.9 \%$ incidence of APL among a total of 801 patients under 18 years of age who were diagnosed with AML in 16 tertiary care medical centers in Korea, during the period between January 2009 and December 2016. In this case, the median age at diagnosis was 10.6 years

Andrade et al. ${ }^{25}$ obtained incidence data in the Brazilian population between 0 and 19 years of age from two different sources. The first was extracted from the databases of 15 Population-Based Cancer Registries during the period 2000-2009 and resulted in a 4.3\% incidence of APL among 805 children and adolescents who were diagnosed with AML. However, the authors estimated that these registries only cover about $20 \%$ of the country's population.

The second set of data was obtained from the records of 33 cancer centers and the Pediatric Hemato-oncology Program of the National Cancer Institute of that country, which covered from 2002 to 2018. 163 cases of APL were characterized (17.5\%) of a total of 931 cases of AML that presented a median age at diagnosis of 10.1 years. ${ }^{25}$ In both cases, they found a higher incidence in southern Brazil, compared to the other geographic regions of the country; which could 
be associated with exposure to environmental factors, since the main economic activities in that area are agriculture and the oil and gas industry. ${ }^{25}$

Aksu et al. ${ }^{8}$ reported an incidence of $20.5 \%$ among a total of 83 patients under 18 years of age who were diagnosed with AML in a hospital in Turkey, between January 2006 and December 2016, with a median of age at diagnosis of 13.5 years.

Czogala et al. ${ }^{9}$ observed that in Poland 41 children under 18 years of age were diagnosed with APL out of a total of 627 patients in that age range who were diagnosed with AML in the period between January 2005 and December 2018, for an incidence of 6.5\%.

\section{Differentiation syndrome (DS)}

This is a syndrome unique to APL, which occurs after ATRA treatment in $20 \%$ of children and can be fatal. In DS, an inflammatory response occurs mediated by an ATRA-induced increase in promyelocyte maturation and cytokine expression. ${ }^{6,26}$

It was described in 1992 by Frankel et al, in 25\% of patients recently diagnosed with APL. It occurs between the 10th and 12th day, from the beginning of the treatment. ${ }^{4,5}$

Symptoms include weight gain, breathing problems, hypotension, and even kidney failure. In $80 \%$ of patients, there is fever and respiratory distress, pulmonary infiltrate, pleural or pericardial effusion. To diagnose the syndrome, at least 3 symptoms must be present. Although the etiology of the syndrome is not defined, infiltration of different organs by myeloid cells is an invariable finding. This occurs due to the excessive amount of maturing myelocytes that causes endothelial damage and edema. It is known that the highest risk of developing this syndrome occurs when the white blood cell counts are initially high, although it has also been observed with low white blood cells. ${ }^{4,6}$

The manifestations of DS caused by ATRA are secondary to three pathophysiological mechanisms: 1) systemic inflammatory response, 2) endothelial damage and microcirculation obstruction, 3) tissue infiltration, due to the release of interleukins, proteases, and expression of endothelial adhesion molecules. ${ }^{6}$ To avoid the complications of DS, hydroxyurea, and steroids should be administered as soon as this pathology is suspected. ${ }^{6}$

\section{Case reports}

Some authors have described in recent years case reports in pediatric patients with APL, which for some reason, are described by some type of singularity. Araus et al. ${ }^{27}$ reported a case of an acute relapse of APL with subcutaneous involvement due to myeloid sarcoma. The patient was a 9-year-old male from Brazil, who presented fever, progressive fatigue, signs of petechiae, and bleeding in the oral cavity. A bone marrow aspirate was performed that showed hypercellularity with granular promyelocytes with Auer bodies, as well as a positive expression of CD33, CD34, CD117 and negative for HLA-DR, positive for the $\mathrm{t}(15 ; 17)$ (q22; q21 translocation) and the fusion between the PML gene and the retinoic acid receptor alpha gene. Daunorubicin and ATRA were given until remission was achieved. Six months later, the patient was readmitted with a recurrence of symptoms, with the presence of a violaceous lesion on the scalp, gingival bleeding, fatigue, fever, and pain in the extremities. Within the laboratory tests, a bone marrow aspirate was performed, which showed $20 \%$ of hypergranular promyelocytes with Auer bodies. Histopathological examination revealed a pattern of large cells and a diffuse infiltrate that also compromised the dermis. The immunohistochemical results were consistent with a myeloid sarcoma in the skin, so it was determined that it was a relapse of APL. The clinical case is striking because extramedullary infiltration in children is extremely rare.

Lee et al. ${ }^{26}$ explained a case of a 13-year-old male patient who presented with fever, leukopenia, and thrombocytopenia. The bone marrow aspirate revealed hypercellularity with $80 \%$ abnormal promyelocytes and the PCR was positive for PML-RARa, for which the patient was diagnosed with APL. ATRA ( $45 \mathrm{mg} / \mathrm{m}^{2} /$ day) was administered immediately after the suspected diagnosis of APL, in addition to dexamethasone as prophylaxis. After the induction phase, the patient developed tinnitus, underwent several hearing tests, and no abnormalities were found. ATRA was discontinued. Later, it was used again and it caused tinnitus and headache, and tests showed a hearing loss, the ATRA was stopped again and after weeks, a notable improvement was found. Investigators concluded that it was an ATRA ototoxicity.

A report from Brazil describes the case of an eight-year-old boy with a previous diagnosis of APL that had finished treatment. He presented gingival bleeding, ecchymosis in the lower limbs, and thrombocytopenia. Blasts are present, so a recurrence is presumed, and ATRA administration is started. On the fourth day, he showed facial edema associated with the appearance of a heart murmur, hyperleukocytosis, and fever. The chest X-ray revealed a bilateral perihilar interstitial infiltrate and the echocardiogram revealed a small volume pericardial effusion. It was concluded that the diagnosis corresponded to ATRA syndrome, for which dexamethasone was administered and the patient evolved satisfactorily. ${ }^{4}$

\section{Conclusion}

APL is mainly caused by the $t(15 ; 17)$ translocation and fusion between the PML gene and the retinoic acid receptor alpha gene, resulting in a protein known as PML-RARa, which in turn causes detention in the maturation of promyelocytes. Even in the absence of $t(15 ; 17)$, rearrangements can occur leading to the production of PML-RARa transcripts. In APL, hemorrhagic and thrombotic events are observed, in fact hemorrhages in the central nervous system and the lungs are the main cause of premature death.

ATRA combined with anthracycline-based chemotherapy is the standard treatment. On the other hand, ATO is being used as a firstline treatment, reaching $86 \%$ effectiveness. However, resistance to both treatments has been observed, which may be due to genetic mutations due to amino acid substitution in the RARp ligand-binding domain (LBD) and the PML-B2 domain of PML-RARA. Another problem associated with ATRA is that it can lead to Differentiation Syndrome, present only in APL. Patients with APL generally have a good outcome, but many of the studies are carried out only in the adult population, so analysis should continue in the pediatric population.

\section{Acknowledgments}

None.

\section{Conflicts of interest}

The authors declare no conflicts of interest.

\section{References}

1. De Albuquerque-Antunes A, Monteiro-Breviglieri CN, Martins-Celeste $\mathrm{D}$, et al. Prevalence and outcomes of thrombotic and hemorrhagic complications in pediatric acute promyelocytic leukemia in a tertiary Brazilian center. Hematol Transfus Cell Ther. 2021;43(3):309-312. 
2. Pardo-Gonzalez CA, Lagos-Ibarra JJ, Ballesteros-Linares A, et al. Results of the implementation of the PETHEMA LPA 99 for treating children with acute promyelocytic leukemia in Bogotá, Colombia. Rev Fac Med. 2021;69(2):e202.

3. Testi AM, Pession A, Diverio D, et al. Risk-adapted treatment of acute promyelicytic leukemia: results from the International Consortium for Childhood APL. Blood. 2018;132(4):405-412.

4. De Paula-Tiago V, Alves-Cunali VC. Síndrome de diferenciación en la leucemia promielocítica: reporte de un caso pediátrico en el HC-UFTM. Residência Pediátrica. 2015;5(3):135-138.

5. Silva-Araujo N, Dos Santos Junior CJ, Da Silva-Gomes V, et al. A Rare Case of Relapsed Pediatric Acute Promyelocytic Leukemia with Skin Involvement by Myeloid Sarcoma. American Journal of Case Reports. 2018;19:438-441.

6. Conneely SE, Stevens A. Advances in Pediatric Acute Promyelocytic Leukemia. Children. 2020;7(11).

7. Noguera N, Catalano G, Banella C, et al. Acute Promyelocytic Leukemia: Update on Mechanisms of Leukemogenesis, Resistance adn on Innovative Treatment Strategies. Cancers. 2019;11:1591.

8. Aksu T, Fettah A, Bozkaya I, et al. Acute Promyelocytic Leukemia in Children: A Single Centre Experience from Turkey. Mediterr J of Hematol and Infect Dis. 2018;10(1):e2018045.

9. Czogala M, Pawinska-Wasikowska K, Ksiazek T, et al. Treatment Outcome and Genetic Characteristics of Acute Promyelocytic Leukemia in Children in Poland From 2005 to 2018. Fontiers in Pediatrics. 2020;8(86)

10. Osumi T, Tsujimoto S, Tamura M, et al. Recurrent RARB translocations in Acute Promyelocytic Leukemia lacking RARA translocation. Cancer Res. 2018;78(16)

11. De Azevedo A, Matsuda E, Cervellini J, et al. Early Mortality in Children and Adolescents with Acute Promyelocytic Leukemia: Experience of the Boldrini Children`s Center. J Pediatr Hematol Oncol. 2019;1-6.

12. Masetti R, Vendemini F, Zama D, et al. All-trans retinoic acid in the treatment of pediatric acute promyelocytic leukemia. Expert Rev Anticancer Ther. 2012;12(9):1191-1204.

13. Moran L, DeanaA,Alonso C. Recaída molecular en leucemia promielocítica aguda. A propósito de un caso. Hematología. 2017;21(1):82-85.

14. Gurnari C, Voso MT, Girardi K, et al. Acute Promyelocytic Leukemia in Children: A Model of Precision Medicine and Chemotherapy-Free Therapy. Int J Mol Sci. 2021;22:642.

15. Park KM, Yoo KH, Kim SK, et al. Clinical Characteristics and Treatment Outcomes of Childhood Acute Promyelocytic Leukemia in Korea: a Nationwide Multicenter Retrospective Study by Korean Pediatric Oncology Study Group. Cancer Res Treat. 2021
16. Sanz MA, Fenaux P, Tallman MS, et al. Management of acute promyelocytic leukemia: updated recommendations from an expert panel of the European Leukemia Net. Blood. 2019;133(15):1630-1643.

17. Kutny MA, Alonzo TA, Gerbing RB, et al. Arsenic Trioxide Consolidation Allows Anthracycline Dose Reduction for Pediatric Patients With Acute Promyelocytic Leukemia: Report From the Children's Oncology Group Phase III Historically Controlled Trial AAML0631. J Clin Oncol. 2017;35: 3021-3029.

18. Zhang L, Zou Y, Chen Y, et al. Role of cytarabine in paediatric acute promyelocytic leukemia treated with the combination of all-trans retinoic acid and arsenic trioxide: a randomized controlled trial. BMC Cancer. 2018; $18: 374$.

19. Kutny MA, Geyer S, Laumann KM, et al. Outcome for Pediatric Acute Promyelocytic Leukemia Patients at Children's Oncology Group Sites on the Leukemia Intergroup Study CALGB 9710 (Alliance). Pediatr Blood Cancer. 2019;66(3):e27542.

20. Hassibe C, Aguilar G, Pereira-Martins D, et al. NTAL is associated with treatment outcome, cell proliferation and differentiation in acute promyelocytic leukemia. Nature Research Scientific Reports. 2020;10:10315.

21. Yamamoto S, Tomizawa D, Kudo K, et al. Hematopoietic stem cell transplantation for pediatric acute promyelocytic leukemia in Japan. Pediatr Blood Cancer. 2019.

22. Yang MH, Wan WQ, Luo JS, et al. Multicenter randomized trial of arsenic trioxide and Realgar-Indigo naturalis formula in pediatric patients with acute promyelocytic leukemia: Interim results of the SCCLG-APL clinical study. Am J Hematol. 2018;93(12):1467-1473.

23. Zheng H, Jiang H, Hu S, et al. Arsenic Combined With All-Trans Retinoic Acid for Pediatric Acute Promyelocytic Leukemia: Report From the CCLG-APL2016 Protocol Study. J Clin Oncol. 2021;39(28):JCO2003096.

24. Jastaniah W, Alsultan A, Al Daama S, et al. Clinical characteristics and outcome of childhood acute promyelocytic leukemia (APL) in Saudi Arabia: a multicenter SAPHOS leukemia group study. Hematology. 2018;23:16-323.

25. Andrade FG, Feliciano SMV, Sardou-Cezar I, et al. Pediatric Acute Promyelocytic Leukemia: Epidemiology, Molecular Features, and Importance of GST-Theta 1 in Chemotherapy Response and Outcome. Front Oncol. 2021;11:642744.

26. Lee JH, Lee JH, Lee JM. All-trans Retinoic Acid-Induced Ototoxicity during Chemotherapy in Pediatric Acute Promyelocytic Leukemia. Children. 2021;8(1):27.

27. Araus NS, Dos Santos Junior CJ, Da Silva-Gomes VM, et al. A Rare Case of Relapsed Pediatric Acute Promyelocytic Leukemia with Skin Involvement by Myeloid Sarcoma. Am J Case Rep. 2018;19:438-441. 